\title{
RELATIVE ASYMPTOTICS FOR ORTHOGONAL MATRIX POLYNOMIALS WITH RESPECT TO A PERTURBED MATRIX MEASURE ON THE UNIT CIRCLE
}

\author{
Hossain O. Yakhlef \\ (Universidad de Granada, Spain) \\ and \\ Francisco Marcellán \\ (Universidad Carlos I De Madrid, Spain)
}

\begin{abstract}
Given a positive definite matrix measure $\Omega$ supported on the unit circle $T$, then main purpose of this paper is to study the asymptotic behavior of $L_{x}(\widetilde{\Omega}) L_{n}(\Omega)^{-1}$ and $\Phi_{x}(z ; \Omega) \Phi_{n}\left(z_{3} \Omega\right)^{-1}$ where

$$
\widehat{\Omega}(z)=\Omega(z)+\mathscr{M} \delta(z-w) ;|w|>1, \mathscr{K}
$$

is a positive definite matrix and $\delta$ is the Dirac matrix measure. Here, $L_{\mathbf{x}}(\cdot)$ means the leading coefficient of the orthonormal matrix polynomials $\Phi_{n}(z ; \cdot)$.

Finally, we deduce the asymptotic behavior of $\Phi_{n}(w ; \Omega) \Phi_{n}(w ; \Omega) \cdot$ in the case when $\mathscr{M}=I$.
\end{abstract}

\section{Introduction}

The theory of matrix polynomials orthogonal with respect to a matrix measure supported on the unit circle appears as a natural tool in many problems of mathematical physics $^{[1]}$, circuits and systems theory ${ }^{[2]}$, linear algebra (in particular, the factorization of positive definite block-Toeplitz matrices, [14]), linear prediction and autoregressive mod$\mathrm{els}^{[7]}$, spectral operator theory ${ }^{[4]}$ among others. 
Particular examples of matrix orthogonal polynomials are connected with scalar orthogonal polynomials associated with positive Borel measures supported on lemniscates, $\mathrm{i}$. e. , algebraic curves of equation $|A(z)|=R$, where $A(z)$ is an algebraic polynomial and $R$ is a nonnegative real number ${ }^{[6]}$.

For a basic approach to the subject, $[1],[2],[3]$ are probably the best references. A classical presentation of matrix measures is [10]. Concerning the distribution of zeros of matrix orthogonal polynomials see [9]. More recently, since the increasing activity around the analytic theory of orthogonal polynomials with respect to measures supported on the unit circle (see $[8],[11]$ ), an analysis of the extension to the matrix case started. A first result in such a direction is [12] where a characterization of reflection matrix parameters is given in terms of a weak asymptotic property of matrix orthogonal polynomials. Furthermore if the determinant of the derivative of the matrix absolutely continuous part of the matrix measure is nonnegative almost everywhere, then the reflection matrix parameters converge to zero. This is the matrix analog of a key result by $E$. Rakhmanov in the scalar case. The importance of such a result is that a new direction can be developed taking into account a general family of matrix measures.

The aim of our contribution is the study of relative asymptotics of two sequences of matrix polynomials orthogonal with respect to two matrix measures whose difference is an atomic matrix measure supported in a point $w$ exterior to the unit circle. We assume that both measures belong to the Szego class and thus the relative outer asymptotics is deduced (Theorem 4.1). Compare with [13] where an analog question is solved on the real line.

The structure of this paper is the following. In section 2 we will introduce the basic properties of orthogonal matrix polynomials on the unit circle. Section 3 is devoted to the relationship between the above mentioned sequences of orthogonal matrix polynomials when a perturbation of the matrix measure via the addition of a matrix mass point is introduced. Here we do not impose any restriction to our initial measure. In such a way, an algebraic approach gives the explicit expressions for the new orthonormal polynomials as well as their leading coefficients. The limit of the ratio of such leading coefficients when the measures belong to the Szego class is obtained (Theorem 3. 4). Finally, in section 4 we prove our main result concerning the relative outer asymptotics for these two sequences of matrix orthonormal polynomials. Notice that it is a natural extension of the scalar case but the proof is very technical and the tools are more sophisticated. 


\section{Orthogonal Matrix Polynomials on the Unit Circle}

Given a $p \times p$ hermitian positive definite matrix-valued measure $\Omega$ on the complex plane $C$, and supported in the unit circle $T=\{z \in C:|z|=1\}$, orthogonal matrix polynomials with respect to $\Omega$ are defined by

$$
\left\langle\Phi_{n}(z ; \Omega), \Phi_{m}(z ; \Omega)\right\rangle_{L} \stackrel{\text { def }}{-} \int_{T} \Phi_{n}(z ; \Omega) \mathrm{d} \Omega \Phi_{m}(z ; \Omega)^{*}=\delta_{n, m}, \quad n, m=0,1,2, \cdots,
$$

or,

$$
\left\langle\Psi_{n}(z ; \Omega), \Psi_{m}(z ; \Omega)\right\rangle_{R} \stackrel{\operatorname{def}}{=} \int_{T} \Psi_{n}(z ; \Omega) * \mathrm{~d} \Omega \Psi_{m}(z ; \Omega)=\delta_{n, m} I, \quad n, m=0,1,2, \cdots,
$$

where

$$
\begin{aligned}
& \Phi_{n}(z ; \Omega)=L_{n}(\Omega) z^{n}+\text { lower degree terms, } \\
& \Psi_{n}(z ; \Omega)=R_{n}(\Omega) z^{n}+\text { lower degree terms, }
\end{aligned}
$$

$I$ is the unit diagonal matrix of dimension $p$ and $L_{n}(\Omega), R_{n}(\Omega)$ are non-singular $p \times p$ matrices. The matrix polynomials $\Phi_{n}(z ; \Omega)$ (resp. $\Psi_{n}(z ; \Omega)$ ) are called left (resp. right) orthonormal matrix polynomials and they are uniquely determined up to the multiplication by a unitary matrix on the left (resp. right). Generally speaking, orthonormal matrix polynomials on the unit circle are determined by the orthonormality relations up to a unitary matrix factor. If we require the relations

$$
\begin{array}{ll}
L_{n}(\Omega)>0, & L_{n}(\Omega)=L_{n}(\Omega)^{*}, \\
R_{n}(\Omega)>0, & R_{n}(\Omega)=R_{n}(\Omega)^{*},
\end{array}
$$

then the orthonormal matrix polynomials are uniquely determined (see [1, page 333$]$ ). Here and in the following, $\Omega$ is a probability matrix measure supported on $T$, if $f$ is a function on $\mathrm{T}$, then $\int_{\mathrm{T}} f \mathrm{~d} \Omega \frac{\text { def }}{2 \pi} \frac{1}{2 \pi} \int_{0}^{2 \pi} f\left(e^{i \theta}\right) \mathrm{d} \Omega(\theta)$. We denote by

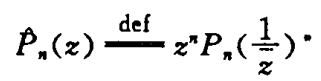

the reversed matrix polynomial, where $A^{*}$ means the conjugate transpose of the matrix $A$.

Let $\left\{C_{k}\right\}_{k=-\infty}^{+\infty}$ be the matrix Fourier coefficients associated with $\Omega$, i. e.,

$$
C_{s} \stackrel{\text { def }}{=} \int_{\mathrm{T}} z^{\mathrm{s}} \mathrm{d} \Omega(z), \quad s \in \mathrm{Z} .
$$

Since $\Omega$ is hermitian, it follows that $C_{-s}=C_{s}^{*}$, and a square block-Toeplitz matrix $\Gamma_{k}(\Omega)$ of order $p(k+1)$ can be constructed as follows 


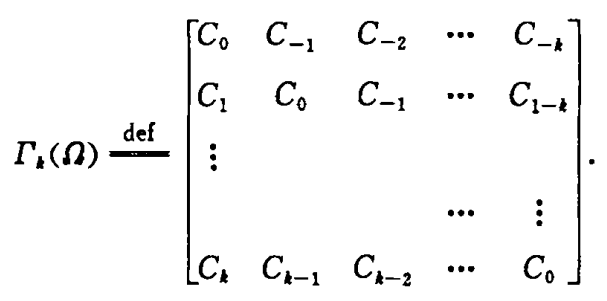

From the properties of $\Omega$ one deduces that $\Gamma_{k}(\Omega)$ is a positive definite matrix for every $k \epsilon$ N.

It is shown that the orthonormal matrix polynomials $\Phi_{n}(z ; \Omega)$ and $\Psi_{n}(z ; \Omega)$ can be constructed as follows (see [2])

$$
\begin{array}{ll}
\Phi_{n}(z ; \Omega)=z^{n} M_{n} A_{n}\left(\frac{1}{\bar{z}}\right)^{*}, & \text { with } M_{n}^{*} M_{n}=A_{n}(0)^{-1}, \\
\Psi_{n}(z ; \Omega)=z^{n} B_{n}\left(\frac{1}{z}\right)^{*} N_{n}, & \text { with } N_{n} N_{n}^{*}=B_{n}(0)^{-1},
\end{array}
$$

where

$$
\left\{\begin{array}{l}
A_{n}(z)=U_{n}(z) \Gamma_{n}^{-1}(\Omega) U_{n}(0)^{T}, \\
B_{n}(z)=V_{n}(0) \Gamma_{n}^{-1}(\Omega) V_{n}(z)^{T}, \\
U_{n}(z)=\left[I, z I, z^{2} I, \cdots, z^{n} I\right], \\
V_{n}(z)=z^{n} U_{n}\left(\frac{1}{z}\right),
\end{array}\right.
$$

and

$$
\begin{aligned}
& A_{n}(z)=A_{n, n} z^{n}+\text { lower degree terms, } \\
& B_{n}(z)=B_{n, n} z^{n}+\text { lower degree terms }
\end{aligned}
$$

are linked through the following relations

$$
\begin{aligned}
& A_{n}(z)-z^{n} B_{n}\left(\frac{1}{\bar{z}}\right)^{*} B_{n}(0)^{-1} A_{n, n}=A_{n-1}(z), \\
& B_{n}(z)-z^{n} B_{n, n} A_{n}(0)^{-1} A_{n}\left(\frac{1}{z}\right)^{*}=B_{n-1}(z),
\end{aligned}
$$

(for an alternative development see $[3]$ and $[14])$. Using (2.3), (2.4), (2.5) and (2.6), the orthonormal matrix polynomials $\Phi_{n}(z ; \Omega)$ and $\Psi_{n}(z, \Omega)$ satisfy

$$
\begin{aligned}
& \Phi_{n}(z ; \Omega)-E_{n} \hat{\Psi}_{n}(z ; \Omega)=z\left(I-E_{n} E_{n}^{*}\right)^{\frac{1}{2}} \Phi_{n-1}(z ; \Omega), \\
& \Psi_{n}(z ; \Omega)-\Phi_{n}(z ; \Omega) E_{n}=z \Psi_{n-1}(z ; \Omega)\left(I-E_{n}^{*} E_{n}\right)^{\frac{1}{2}},
\end{aligned}
$$

where

$$
E_{n}=M_{n} A_{n n}^{*} N_{n}=M_{n} B_{n n}^{*} N_{n}
$$

are called the reflection coefficients and $I-E_{n} E_{n}^{*}=M_{n} M_{n-1}^{-1} M_{n-1}^{-1 *} M_{n}^{*}$, which shows that $I$ 
$-E_{n} E_{n}^{*}$ is positive definite or $E_{n} E_{n}^{*}<I$ (compare this fact with the scalar case). Combining (2.7) and (2.8) and taking into account the identity $\left(I-E_{n} E_{n}^{*}\right)^{\frac{1}{2}} E_{n}=E_{n}\left(I-E_{n}^{*} E_{n}\right)^{\frac{1}{2}}$ we get the following recurrence relations

$$
\begin{aligned}
& \left(I-E_{n} E_{n}^{*}\right)^{\frac{1}{2}} \Phi_{n}(z ; \Omega)=z \Phi_{n-1}(z ; \Omega)+E_{n} \hat{\Psi}_{n-1}(z ; \Omega), \\
& \Psi_{n}(z ; \Omega)\left(I-E_{n}^{*} E_{n}\right)^{\frac{1}{2}}=z \Psi_{n-1}(z ; \Omega)+\Phi_{n-1}(z ; \mathrm{d} \Omega) E_{n} .
\end{aligned}
$$

Notice that for any matrix measure $\Omega$, the matrix parameters $E_{n}$ are uniquely determined, up to left and right unitary factors independent of $n$. For the converse result (Favard's theorem for orthogonal matrix polynomials on the unit circle) see [2].

The matrix version of the Christoffel-Darboux formula appears in the literature in several forms ${ }^{[14]}$. Using (2.10), (2.11) and the identity $\left(I-E_{n} E_{n}^{*}\right)^{-1} E_{n}=E_{n}(I-$ $\left.E_{n}^{*} E_{n}\right)^{-1}$, we can find

$$
\begin{aligned}
& \Phi_{k}(z ; \Omega) \Phi_{k}(\xi ; \Omega)-\xi \Phi_{k-1}(z ; \Omega) \Phi_{k-1}(\xi ; \Omega) \\
& =\Psi_{k}(z ; \Omega) \hat{\Psi}_{k}(\xi ; \Omega)-z \Psi_{k-1}(z ; \Omega) \hat{\Psi}_{k-1}(\xi ; \Omega) .
\end{aligned}
$$

Multiplying both sides of (2.12) by $z^{-k}$, adding up for $k=0,1, \cdots, n$ and taking into account (2.2), we get

$$
z^{-n} \Psi_{n}(z ; \Omega) \Psi_{n}(\xi ; \Omega)=\left(1-\frac{\xi}{z}\right) \sum_{k=0}^{n} \Phi_{k}\left(\frac{1}{\bar{z}} ; \Omega\right){ }^{*} \Phi_{k}(\xi ; \Omega)+\frac{\xi}{z} \Phi_{n}\left(\frac{1}{\bar{z}} ; \Omega\right){ }^{*} \Phi_{n}(\xi ; \Omega) .
$$

Replacing $z$ by $\frac{1}{\bar{z}}$, we get the Christoffel-Darboux formula

$$
\begin{aligned}
& (1-\bar{z} \xi) K_{n}(z ; \xi ; \Omega) \frac{\text { def }}{2}(1-\bar{z} \xi) \sum_{k=0}^{n} \Phi_{k}(z ; \Omega) * \Phi_{k}(\xi ; \Omega) \\
& =\hat{\Psi}_{n}(z ; \Omega)^{*} \hat{\Psi}_{n}(\xi ; \Omega)-\bar{z} \xi \Phi_{n}(z ; \Omega)^{*} \Phi_{n}(\xi ; \Omega) .
\end{aligned}
$$

In a similar way we can obtain the dual formula

$$
\begin{aligned}
& (1-\bar{z} \xi) \mathscr{X}_{n}(z, \xi ; \Omega) \frac{\text { def }}{}(1-\bar{z} \xi) \sum_{k=0}^{n} \Psi_{k}(\xi ; \Omega) \Psi_{k}(z ; \Omega) \text { * } \\
& =\Phi_{n}(\xi ; \Omega) \Phi_{n}(z ; \Omega)^{*}-\bar{z} \xi \Psi_{n}(\xi ; \Omega) \Psi_{n}(z ; \Omega)^{*} .
\end{aligned}
$$

Setting $\xi=z$ in $(2.13)$ one has

$$
|z|^{2} \Phi_{n}(z ; \Omega) * \Phi_{n}(z ; \Omega)=\left(|z|^{2}-1\right) \mathscr{K}_{n}(z, z ; \Omega)+\hat{\Psi}_{n}(z ; \Omega){ }^{*} \Psi_{n}(z ; \Omega) .
$$

It is clear that the right hand side of $(2.15)$ is positive definite for $|z|>1$, then $\Phi_{n}(z ; \Omega)$ is non-singular outside the closed unit disk. Using (2.15) and (2.7), one can show that $\Phi_{n}(z, \Omega)$ could not have any zero when $|z|=1\left(\Phi_{n}(z ; \Omega)\right.$ is said to have a zero at the point $z=z_{0}$ if $\Phi_{n}\left(z_{0} ; \Omega\right)$ is non-singular, i. e., $\left.\operatorname{det} \Phi_{n}\left(z_{0}, \Omega\right)=0\right)$. Hence the zeros of $\Phi_{n}(z, \Omega)$ lie on the open unit disk. In a similar way, it is possible to obtain the same result for $\Psi_{n}(z$; $\Omega)$ using (2.14) and (2.8). It is worth observing that the matrix polynomials $\mathscr{K}_{n}(z, \xi$; 
$\Omega)$ and $\mathscr{T}_{n}(z, \xi, \Omega)$ satisfy the following property

$$
\begin{array}{lll}
\left\langle\Pi_{m}(\xi), \mathscr{K}_{n}(z, \xi ; \Omega)\right\rangle_{L}=\Pi_{m}(z) ; & & m \leqslant n, \\
\left\langle\mathscr{X}_{n}(z, \xi, \Omega), \Pi_{m}(\xi)\right\rangle_{R}=\Pi_{m}(z), & & m \leqslant n,
\end{array}
$$

where $\Pi_{m}(z)$ is any matrix polynomial of degree $m$.

In fact, writing $\Pi_{m}(\xi)=\sum_{r=0}^{*} A_{r} \Phi_{r}(\xi ; \Omega)$, we have

$$
\begin{aligned}
\left\langle\Pi_{m}(\xi), \mathscr{K}_{n}(z, \xi, \Omega)\right\rangle_{L} & =\int_{T} \Pi_{m}(\xi) \mathrm{d} \Omega(\xi) \mathscr{K}_{n}(z, \xi ; \Omega)^{*} \\
& =\sum_{r=0}^{m} A_{r} \sum_{k=0}^{n} \int_{T} \Phi_{r}(\xi ; \Omega) \mathrm{d} \Omega(\xi) \Phi_{k}(\xi ; \Omega)^{*} \Phi_{k}(z ; \Omega) \\
& =\sum_{r=0}^{m} A_{r} \Phi_{r}(z ; \Omega)=\Pi_{m}(z) .
\end{aligned}
$$

Now, writing $\Pi_{m}(\xi)=\sum_{r=0}^{m} \Psi_{r}(\xi, \Omega) B_{r}$, then

$$
\begin{aligned}
\left\langle\mathscr{C}_{n}(z, \xi ; \Omega), \Pi_{m}(\xi)\right\rangle_{R} & =\int_{T} \mathscr{E}_{n}(z, \xi ; \Omega) \cdot \mathrm{d} \Omega(\xi) \Pi_{m}(\xi) \\
& =\sum_{r=0}^{m} \sum_{k=0}^{n} \Psi_{k}(z ; \Omega) \int_{T} \Psi_{k}(\xi ; \Omega) \cdot \mathrm{d} \Omega(\xi) \Psi_{r}(\xi ; \Omega) B_{r} \\
& =\sum_{r=0}^{m} \Psi_{r}(z ; \Omega) B_{r}=\Pi_{m}(z) .
\end{aligned}
$$

Necessary and sufficient conditions for the convergence of orthogonal matrix polynomials on the unit circle have been derived in several forms (see $[2$, Thms 18,19]), and deal with the case when

$$
\begin{aligned}
\log \operatorname{det} W \in L^{1}(T) & \Leftrightarrow \sum_{k=1}^{\infty}\left\|E_{k}\right\|_{E}^{2}<\infty \\
& \Leftrightarrow \sup _{k \in N}\left[\operatorname{det} A_{k}(0)\right]<\infty,
\end{aligned}
$$

where $W(\theta)=\frac{\mathrm{d} \Omega(\theta)}{\mathrm{d} \theta}$ a. e. denotes the Radon-Nikodym derivative of the absolutely continuous part of the matrix measure $\Omega$ with respect to the Lebesgue scalar measure, and $\|$ • $\|_{E}$ is the Frobenius norm (see [5,page 291]).

Theorem 2. $1^{[2]}$. The sequence $\left\{A_{k}(z)\right\}_{k \in N}$ converges uniformly on every compact subset of $D=\left\{z \in C_{z}|z|<1\right\}$ if and only if the numbers $\operatorname{det} A_{k}(0)$ are bounded from above. In this case, the limit $A(z)$ of $\left\{A_{k}(z)\right\}_{k \in N}$ is an analytic matrix function and has no zeros in $D$; its inverse $A(z)^{-1}$ belongs to the class $H_{2}^{p \times p}$.

It should be pointed out that the dual family $\left\{B_{k}(z)\right\}_{k \in \mathrm{N}}$ has the same type of properties as the family $\left\{A_{k}(z)\right\}_{n \in \mathbb{N}}$. 
In the next sections, we will need the asymptotics of the ratio of the left and right orthonormal matrix polynomials on the unit circle, given in the following Theorem.

Theorem 2. 2. Let $\Omega$ be a hermitian positive definite matrix-valued measure supported on $\mathrm{T}$, and $\left(\Phi_{n}(z ; \Omega)\right)_{n \in \mathbb{N}},\left(\Psi_{n}(z ; \Omega)\right)_{n \in \mathbb{N}}$ be its left and right associated orthonormal matrix polynomials. If the Szegö condition

$$
\log \operatorname{det} W \in L^{1}(T)
$$

holds, then

$$
\begin{aligned}
& \lim _{n \rightarrow \infty} \hat{\Psi}_{n}(w ; \Omega) \Phi_{n}(w ; \Omega)^{-1}=0, \\
& \lim _{n \rightarrow \infty} \Psi_{n}(w ; \Omega)^{-1} \hat{\Phi}_{n}(w ; \Omega)=0,
\end{aligned}
$$

when $w$ is outside the closed unit disk.

Proof. From (2.8) we have

$$
w^{n} \Psi_{n}\left(\frac{1}{\bar{w}} ; \Omega\right)^{*}=E_{n}^{*} \Phi_{n}(w ; \Omega)+\left(I-E_{n}^{*} E_{n}\right)^{\frac{1}{2}} w^{n-1} \Psi_{n-1}\left(\frac{1}{\bar{w}} ; \Omega\right)^{*}
$$

and then

$$
\begin{aligned}
& \Psi_{n}(w ; \Omega) \Phi_{n}(w ; \Omega)^{-1} \\
& =E_{n}^{*}+\left(I-E_{n}^{*} E_{n}\right)^{\frac{1}{2}} \hat{\Psi}_{n-1}(w ; \Omega) \Phi_{n-1}(w ; \Omega)^{-1} \Phi_{n-1}(w ; \Omega) \Phi_{n}(w ; \Omega)^{-1} .
\end{aligned}
$$

But from (2.3)

$$
\Phi_{n-1}(w ; \Omega) \Phi_{n}(w ; \Omega)^{-1}=\frac{1}{w} M_{n-1} A_{n-1}\left(\frac{1}{w}\right) \cdot A_{n}\left(\frac{1}{w}\right)^{-*} M_{n}^{-1},
$$

and using Theorem 2.1 and (2.3), we get

$$
\lim _{n \rightarrow \infty} \Phi_{n-1}(w ; \Omega) \Phi_{n}(w ; \Omega)^{-1}=\frac{1}{w} I, \quad|w|>1 .
$$

Let $\varepsilon_{1}$ be a non-negative real number $\left(\varepsilon_{1}+\frac{1}{|w|}:=\rho<1\right)$, then there exists a positive integer number $N_{1}$ such that for every $n \geqslant N_{1}$,

$$
\left|\left\|\Phi_{n-1}(w ; \Omega) \Phi_{n}(w ; \Omega)^{-1}\right\|_{2}-\left\|\frac{1}{w} I\right\|_{2}\right| \leqslant\left\|\Phi_{n-1}(w ; \Omega) \Phi_{n}(w ; \Omega)^{-1}-\frac{1}{w} I\right\|_{2}<\varepsilon_{1},
$$

where $\|\cdot\|_{2}$ is the spectral norm (see $[5$, page 295]).

From (2.9) we have $E_{n} E_{n}^{*} \leqslant I$, and then

$$
\left\|\left(I-E_{n}^{*} E_{n}\right)^{\frac{1}{2}}\right\|_{2} \leqslant 1
$$

for all $n \in N$.

Using (2.22), (2.24) and (2.25) we get there exists $N_{2} \geqslant N_{1}$, such that for every $n$ $\geqslant N_{2}$,

$$
\left\|\Psi_{n}(w ; \Omega) \Phi_{n}(w ; \Omega)^{-1}\right\|_{2} \leqslant\left\|E_{n}\right\|_{2}+
$$




$$
\left(\varepsilon_{1}+\frac{1}{|w|}\right)\left\|\Psi_{n-1}\left(w_{i} \Omega\right) \Phi_{n-1}(w ; \Omega)^{-1}\right\|_{2} .
$$

Notice that $\left(\hat{\Psi}_{n}(w, \Omega) \Phi_{n}(w, \Omega)^{-1}\right)_{n \in N}$ verifies the followign inequality for all $n$ and $k$ such that $n-k \geqslant N_{2}$,

$$
\begin{aligned}
& \left\|\hat{\Psi}_{n}(w ; \Omega) \Phi_{n}(w, \Omega)^{-1}\right\|_{2} \\
& \quad \leqslant \sum_{i=n-k+1}^{n} \rho^{n-i}\left\|E_{i}\right\|_{2}+\rho^{k}\left\|\Psi_{n-k}(w ; \Omega) \Phi_{n-k}\left(w_{3} \Omega\right)^{-1}\right\|_{2}
\end{aligned}
$$

In fact, if $k=1$,

$$
\left\|\Psi_{n}(w ; \Omega) \Phi_{n}(w ; \Omega)^{-1}\right\|_{2} \leqslant\left\|E_{n}\right\|_{2}+\rho\left\|\hat{\Psi}_{n-1}(w ; \Omega) \Phi_{n-1}(w ; \Omega)^{-1}\right\|_{2},
$$

which is true from (2.26). We suppose that (2.27) is true for $k \in N\left(n-k \geqslant N_{2}\right)$. Then from (2.27) and replacing $n$ by $n-k$ in (2.26), we get

$$
\begin{aligned}
\left\|\hat{\Psi}_{n}(w ; \Omega) \Phi_{n}(w ; \Omega)^{-1}\right\|_{2} \leqslant & \sum_{i=n-k+1}^{n} \rho^{n-i}\left\|E_{i}\right\|_{2}+ \\
& \rho^{k}\left(\left\|E_{n-k}\right\|_{2}+\rho\left\|\Psi_{n-k-1}(w ; \Omega) \Phi_{n-k-1}(w ; \Omega)^{-1}\right\|_{2}\right) \\
= & \sum_{i=n-k}^{n} \rho^{n-i}\left\|E_{i}\right\|_{2}+ \\
& \rho^{k+1}\left\|\Psi_{n-(k+1)}(w ; \Omega) \Phi_{n-(k+1)}(w ; \Omega)^{-1}\right\|_{2} .
\end{aligned}
$$

Thus, by induction we deduce (2.27).

Now, we suppose that the matrix $\Psi_{n}\left(w_{3} \Omega\right) \Phi_{n}\left(w_{3} \Omega\right)^{-1}$ does not converge to 0 , then given a positive real number $\varepsilon_{2}$ there exists a positive integer $N_{4}$ such that for every $m>$ $N_{4}$

$$
\varepsilon_{2} \leqslant\left\|\Psi_{m}(w ; \Omega) \Phi_{m}\left(w_{i} \Omega\right)^{-1}\right\|_{2} .
$$

Therefore, using (2.29), we can construct a subsequence $\left(\Psi_{\left.\alpha_{n}\right)}\left(w_{;} \Omega\right) \Phi_{\left.\alpha_{n}\right)}\left(w_{3} \Omega\right)^{-1}\right)_{n \in \mathbb{N}}$ which satisfies

$$
0<\varepsilon_{2} \leqslant\left\|\Psi_{q(n)}(w ; \Omega) \Phi_{(\alpha)}(w ; \Omega)^{-1}\right\|_{2 ;} \quad \forall n \in N .
$$

In fact, if $\Psi_{\rho(k)}(w ; \Omega) \Phi_{\rho(k)}\left(w_{q} \Omega\right)^{-1}$ is given for $k=1,2, \cdots, n$, then for $\varphi(n) \in N$, and from (2.29), there exists a positive integer number $\varphi(n+1)=: m>\varphi(n)$ such that

$$
\varepsilon_{2} \leqslant\left\|\Psi_{\left.\alpha_{n+1}\right)}\left(w_{;} \Omega\right) \Phi_{\alpha n+1)}\left(w_{;} \Omega\right)^{-1}\right\|_{2} .
$$

Thus, by recurrence, we get $\left(\Psi_{(n)}(w ; \Omega) \Phi_{\alpha n)}(w ; \Omega)^{-1}\right)_{n \in N}$ satisfying $(2.30)$.

Since $\varphi(n)$ is an increasing sequence of positive integer numbers, then for each $n \in N$, there exists $k>0$ which depends of $n$, such that $\varphi(n)=\varphi(n-1)+k$. Replacing $n$ by $\varphi(n)$ in (2. 27), one has

$$
\left\|\Psi_{\alpha n)}\left(w_{3} \Omega\right) \Phi_{\left.\alpha_{n}\right)}\left(w_{;} \Omega\right)^{-1}\right\|_{2}
$$




$$
\leqslant \sum_{i=\phi_{(n-1)+1}}^{\alpha(n)} \rho^{(\alpha n)-i}\left\|E_{i}\right\|_{2}+\rho^{\left.\alpha(n)-\alpha_{n}-1\right)}\left\|\hat{\Psi}_{\kappa(n-1)}(w ; \Omega) \Phi_{\alpha(n-1)}(w ; \Omega)^{-1}\right\|_{2 \cdot}(2.31)
$$

for all $n>N_{2}$.

From (2.19), we have $\lim _{n \rightarrow \infty}\left\|E_{n}\right\|_{2}=0$. Then for all $\varepsilon>0$, and for $n$ large enough, we get

$$
\left\|E_{n}\right\|_{2} \leqslant \varepsilon
$$

and

$$
0 \leqslant \sum_{\left.i=\alpha_{n-1}\right)+1}^{\alpha n\}} \rho^{(\alpha n)-i}\left\|E_{i}\right\|_{2} \leqslant \varepsilon \sum_{i=\alpha(n-1)+1}^{\alpha(n)} \rho^{(\alpha)-i} \leqslant \varepsilon \frac{1}{1-\rho} .
$$

Therefore

$$
\lim _{n \rightarrow \infty} \sum_{i=\alpha n-1)+1}^{(n)} \rho^{\alpha(n)-i}\left\|E_{i}\right\|_{2}=0
$$

From (2.31), we have

$$
\begin{aligned}
& \exists N_{2} \geqslant N_{1}, \forall n>N_{2}, \\
& \frac{\left\|\Psi_{\alpha(n)}(w ; \Omega) \Phi_{\alpha(n)}(w ; \Omega)^{-1}\right\|_{2}}{\left\|\Psi_{\alpha(n-1)}(w ; \Omega) \Phi_{\alpha(n-1)}(w ; \Omega)^{-1}\right\|_{2}} \leqslant \frac{\sum_{\left.i=\alpha_{n}-1\right)+1}^{\alpha(n)} \rho^{\alpha(n)-i}\left\|E_{i}\right\|_{2}}{\left\|\Psi_{\alpha_{n-1}}(w ; \Omega) \Phi_{\alpha(n-1)}(w ; \Omega)^{-1}\right\|_{2}}+\rho^{\left.\phi(n)-\alpha_{n-1}\right)} .
\end{aligned}
$$

Hence, using (2.32) and taking into account (2.30), we have

$$
\begin{aligned}
& \exists N_{3} \geqslant N_{2}, \exists \kappa<1, \forall n \geqslant N_{3}, \\
& \left\|\hat{\Psi}_{(n)}(w ; \Omega) \Phi_{\alpha n)}(w ; \Omega)^{-1}\right\|_{2} \leqslant \kappa\left\|\hat{\Psi}_{\alpha(n-1)}(w ; \Omega) \Phi_{\alpha(n-1)}(w ; \Omega)^{-1}\right\|_{2},
\end{aligned}
$$

i.e. ,

$$
\left\|\Psi_{\alpha n)}(w ; \Omega) \Phi_{\alpha n)}\left(w_{;} \Omega\right)^{-1}\right\|_{2} \leqslant \kappa^{n-N_{3}}\left\|\Psi_{\left.\alpha N_{3}\right)}\left(w_{3} \Omega\right) \Phi_{\left.\alpha N_{3}\right)}\left(w_{;} \Omega\right)^{-1}\right\|_{2},
$$

and then

$$
\lim _{n \rightarrow \infty} \Psi_{\alpha(n)}(w ; \Omega) \Phi_{\alpha(n)}(w ; \Omega)^{-1}=0,
$$

which contradicts (2.30). Hence (2.20) holds.

To prove (2.21), we use (2.7). Then we have

$$
\hat{\Phi}_{n}(w ; \Omega)=\Psi_{n}(w ; \Omega) E_{n}^{*}+\hat{\Phi}_{n-1}(w ; \Omega)\left(I-E_{n} E_{n}^{*}\right)^{\frac{1}{2}} .
$$

Therefore

$$
\begin{aligned}
& \Psi_{n}(w ; \Omega)^{-1} \Phi_{n}(w ; \Omega)=E_{n}^{*}+ \\
& \quad\left(\Psi_{n}(w ; \Omega)^{-1} \Psi_{n-1}(w ; \Omega)\right) \Psi_{n-1}(w ; \Omega)^{-1} \Phi_{n-1}(w ; \Omega)\left(I-E_{n} E_{n}^{*}\right)^{\frac{1}{2}}
\end{aligned}
$$

From (2.4) and Theorem 2.1,

$$
\lim _{n \rightarrow \infty} \Psi_{n}(w ; \Omega)^{-1} \Psi_{n-1}(w ; \Omega)=\frac{1}{w} I, \quad|w|>1 .
$$

Then, using the same way as before, we get (2.21). 


\section{Comparison of Orthogonal Matrix Polynomials and Ratio Asymptotics for the Leading Coefficients}

Let $\Omega$ be a hermitian positive definite matrix-valued measure supported on the unit circle $T$, and $\widetilde{\Omega}$ its perturbation by the addition of a Dirac matrix measure at the point $w \in$ C

$$
d \widetilde{\Omega}(z)=d \Omega(z)+\mathscr{A} \delta(z-w)
$$

where $\mathscr{M}$ is a positive definite matrix.

Let $\left(\Phi_{n}(z, .)=L_{n}(.) z^{n}+\cdots\right)_{n \in \mathbb{N}}\left(\operatorname{resp} .\left(\Psi_{n}(z, .)=R_{n}(.) z^{n}+\cdots\right)_{n \in \mathbb{N}}\right)$ be a sequence of left (resp. right) orthonormal matrix polynomials with respect to a matrix measure. Our aim is to investigate the ralationship between two sequence of orthonormal matrix polynomials when the associated matrix measures are $\bar{\Omega}$ and $\Omega$, respectively, and find the limit of the ratio of their leading coefficients.

Lemma 3.1. Let $\Omega$ and $\Omega$ be two matrix measures related by (3.1), then

1. $\Phi_{n}(z ; \widetilde{\Omega})=\left[L_{n}(\Omega)\right]^{-*} L_{n}(\Omega) *\left\{\Phi_{n}(z ; \Omega)-\Phi_{n}(w ; \Omega)[I+\right.$

$$
\left.\left.\mathscr{M} \mathscr{K}_{n}(w, w ; \Omega)\right]^{-1} \mathscr{M} \mathscr{K}_{n}\left(z, w_{n} \Omega\right)^{*}\right\} ;
$$

2. $\Psi_{n}(z ; \widetilde{\Omega})=\left\{\Psi_{n}\left(z ; \Omega-\mathscr{X}_{n}(z, w ; \Omega) * \mathscr{M}[I+\right.\right.$

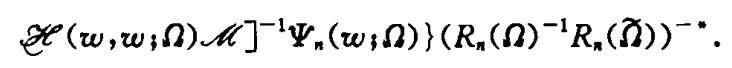

Proof. Using (2.16) and (2.17), we have

$$
\begin{aligned}
\Phi_{n}(z ; \widetilde{\Omega})= & \left\langle\Phi_{n}(\xi ; \widetilde{\Omega}), \mathscr{K}_{n}(z, \xi ; \Omega)\right\rangle_{L} \\
= & \int_{T} \Phi_{n}(\xi ; \widetilde{\Omega}) d \Omega(\xi) \mathscr{K}_{n}(z, \xi ; \Omega)^{*} \\
= & \int_{T \cup\{w\}} \Phi_{n}(\xi ; \widetilde{\Omega}) d \widetilde{\Omega}(\xi) \mathscr{K}_{n}(z, \xi ; \Omega)^{*}- \\
& \int_{(w\}} \Phi_{n}(\xi ; \widetilde{\Omega}) \mathscr{M} \mathscr{K}_{n}(z, \xi ; \Omega) * \delta(\xi-w) \\
= & \int_{T U(w\}} \Phi_{n}(\xi ; \widetilde{\Omega}) d \widetilde{\Omega}(\xi) \Phi_{n}(\xi ; \Omega) * \Phi_{n}(z ; \Omega)-\Phi_{n}(w ; \widetilde{\Omega}) \mathscr{M} \mathscr{K}_{n}(z, w ; \Omega) * \\
= & {\left[L_{n}(\widetilde{\Omega})\right]^{-*} L_{n}(\Omega) * \Phi_{n}(z ; \Omega)-\Phi_{n}(w ; \widetilde{\Omega}) \mathscr{M C}_{n}(z, w ; \Omega) * }
\end{aligned}
$$

and

$$
\begin{aligned}
\Psi_{n}(z ; \widetilde{\Omega}) & =\left\langle\mathscr{Z}_{n}(z, \xi ; \Omega), \Psi_{n}(\xi ; \widetilde{\Omega})\right\rangle_{R} \\
& =\Psi_{n}(z ; \Omega) \int_{T \cup\{w\}} \Psi_{n}(\xi ; \Omega) * d \widetilde{\Omega}(\xi) \Psi_{n}(\xi ; \widetilde{\Omega})-\mathscr{Z}_{n}\left(z, w_{;} \Omega\right) * \mathscr{M}_{n} \Psi_{n}(w ; \widetilde{\Omega}) \\
& =\Psi_{n}(z ; \Omega) R_{n}(\Omega) *\left[R_{n}(\widetilde{\Omega})\right]^{-*}-\mathscr{Z}_{n}(z, w ; \Omega) * \mathscr{M}_{n}\left(w_{;} \widetilde{\Omega}\right) .
\end{aligned}
$$


If $z=w$, then

$$
\begin{aligned}
& \Phi_{n}(w ; \Omega)\left[I+\mathscr{M} \mathscr{K}_{n}(w, w ; \Omega)\right]=\left[L_{n}(\Omega)\right]^{-*} L_{n}(\Omega){ }^{*} \Phi_{n}\left(w_{1} \Omega\right), \\
& {\left[I+\mathscr{X}_{n}(w, w ; \Omega) \mathscr{M}\right] \Psi_{n}\left(w_{3} \Omega\right)=\Psi_{n}(w ; \Omega) R_{n}(\Omega) \cdot\left[R_{n}(\Omega)\right]^{-*} .}
\end{aligned}
$$

Since $\mathscr{M}, \mathscr{K}_{n}(w, w ; \Omega)$ and $\mathscr{X}_{n}\left(w, w_{;} \Omega\right)$ are positive definite matrices, then

$$
\begin{aligned}
& I+\mathscr{M} \mathscr{K}_{n}\left(w, w_{n} \Omega\right)=\mathscr{M}\left(\mathscr{M}^{-1}+\mathscr{K}_{n}\left(w, w_{1} \Omega\right)\right), \\
& I+\mathscr{X}_{n}\left(w, w_{1} \Omega\right) \mathscr{M}=\left(\mathscr{M}^{-1}+\mathscr{K}_{n}\left(w, w_{l} \Omega\right)\right) \mathscr{M}
\end{aligned}
$$

are non-singular, as a product of two positive definite matrices. Hence

$$
\begin{aligned}
& \Phi_{n}\left(w_{3},\right)=\left[L_{n}(\widetilde{\Omega})\right]^{-*} L_{n}(\Omega) * \Phi_{n}\left(w_{;} \Omega\right)\left[I+\mathscr{M} \mathscr{K}_{n}(w, w ; \Omega)\right]^{-1}, \\
& \Psi_{n}\left(w_{3} \Omega\right)=\left[I+\mathscr{K}_{n}\left(w, w_{3} \Omega\right) \mathscr{M}\right]^{-1} \Psi_{n}\left(w_{3} \Omega\right) R_{n}(\Omega) *\left[R_{n}(\widetilde{\Omega})\right]^{-*} .
\end{aligned}
$$

Finally using (3.2), (3.3), (3.4) and (3.5) we get the parts 1 and 2 of Lemma 3.1.

Proposition 3. 2. Let $\widehat{\Omega}$ and $\Omega$ be two matrix measures related by (3.1) where $|w| \geqslant$ 1 , then

1. $\left(L_{n}(\widetilde{\Omega}) L_{n}(\Omega)^{-1}\right)^{*}\left(L_{n}(\widetilde{\Omega}) L_{n}(\Omega)^{-1}\right)=I-\left[\Phi_{n}\left(w_{3} \Omega\right)^{-*} \mathscr{M}^{-1} \Phi_{n}\left(w_{3} \Omega\right)^{-1}+\right.$ $\left.\Phi_{n}(w ; \Omega)^{-\cdot} \mathscr{K}_{n}(w, w ; \Omega) \Phi_{n}(w ; \Omega)^{-1}\right]^{-1}$;

2. $\left(R_{n}(\Omega)^{-1} R_{n}(\Omega)\right)\left(R_{n}(\Omega)^{-1} R_{n}(\Omega)\right)^{*}=I-\left[\Psi_{n}\left(w_{3} \Omega\right)^{-1} \mathscr{M}^{-1} \Psi_{n}\left(w_{3} \Omega\right)^{-*}+\right.$ $\left.\Psi_{n}(w ; \Omega)^{-1} \mathscr{Z}_{n}(w, w ; \Omega) \Psi_{n}(w ; \Omega)^{-*}\right]^{-1}$.

Proof. Using Lemma 3.1,

$$
\begin{aligned}
& \int_{T} \Phi_{n}(z ; \widetilde{\Omega}) d \Omega(z) \Phi_{n}(z ; \Omega) *=\left[L_{n}(\widetilde{\Omega})\right]^{-*} L_{n}(\Omega) * \\
& \quad\left\{I-\Phi_{n}(w ; \Omega)\left[I+\mathscr{M} \mathscr{K}_{k}\left(w, w_{i} \Omega\right)\right]^{-1} \mathscr{A} \int_{T} \mathscr{K}_{n}\left(z, w_{i} \Omega\right)^{*} d \Omega(z) \Phi_{n}(z ; \Omega) *\right\}
\end{aligned}
$$

and

$$
\begin{aligned}
& \int_{T} \Psi_{n}(z ; \Omega) * d \Omega(z) \Psi_{n}(z ; \Omega)=\left\{I-\int_{T} \Psi_{n}(z ; \Omega) * d \Omega(z) \mathscr{X}_{n}\left(z, w_{;} \Omega\right) * \mathscr{A}[I+\right. \\
& \left.\left.\mathscr{X}_{n}(w, w ; \Omega) \mathscr{M}\right]^{-1} \Psi_{n}(w, \Omega)\right\} R_{n}(\Omega) *\left[R_{n}(\Omega)\right]^{-*}
\end{aligned}
$$

Using (2.16) and (2.17) we get

$$
\begin{aligned}
& \left(L_{n}(\Omega) L_{n}(\Omega)^{-1}\right)=\left(L_{n}(\Omega) L_{n}(\Omega)^{-1}\right)^{-*}\{I- \\
& \left.\left.\Phi_{n}(w ; \Omega)\left[I+\mathscr{A} \mathscr{K}_{n}(w, w ; \Omega)\right]^{-1} \mathscr{M} \Phi_{n}(w ; \Omega)^{*}\right\}\right]
\end{aligned}
$$

and

$$
\begin{aligned}
& \left(R_{n}(\Omega)^{-1} R_{n}(\Omega)\right)=\left\{I-\Psi_{n}\left(w_{3} \Omega\right) * \mathscr{M}[I+\right. \\
& \left.\left.\mathscr{E}_{n}\left(w, w_{i} \Omega\right) \mathscr{M}\right]^{-1} \Psi_{n}\left(w_{3} \Omega\right\}\left(R_{n}(\Omega)^{-1} R_{n}(\Omega)\right)^{-*}\right] .
\end{aligned}
$$

Then

$$
\begin{aligned}
& \left(L_{n}(\Omega) L_{n}(\Omega)^{-1}\right) \cdot\left(L_{n}(\Omega) L_{n}(\Omega)^{-1}\right) \\
& \quad=I-\Phi_{n}\left(w_{3} \Omega\right)\left[\Phi_{n}\left(w_{3} \Omega\right)^{-*} \mathscr{M}^{-1}+\Phi_{n}\left(w_{3} \Omega\right)^{-*} \mathscr{K}_{n}\left(w_{,} w_{3} \Omega\right)\right]^{-1}
\end{aligned}
$$


and

$$
\begin{aligned}
& \left(R_{n}(\Omega)^{-1} R_{n}(\Omega)\right)\left(R_{n}(\Omega)^{-1} R_{n}(\Omega)\right)^{*} \\
& \quad=I-\Psi_{n}(w ; \Omega) *\left[\Psi_{n}(w ; \Omega)^{-1} \mathscr{M}^{-1}+\Psi_{n}(w ; \Omega)^{-1} \mathscr{Z}_{n}(w, w ; \Omega)\right]^{-1} .
\end{aligned}
$$

Which yield the statements 1 and 2 .

Remark 3. 3. The matrices $\left(L_{n}(\nwarrow) L_{n}(\Omega)^{-1}\right)^{\cdot}$ and $\left(R_{n}(\Omega)^{-1} R_{n}(\Omega)\right)$ can be assumed to be lower triangular matrices with positive diagonal elements.

Indeed, if the sequences $\left(L_{n}(\Omega) L_{n}(\Omega)^{-1}\right)_{n \in \mathbb{N}}\left(\right.$ resp. $\left(R_{n}(\Omega)^{-1} R_{n}(\Omega)\right)_{n \in N}$ ) are not upper triangular matrices with positive diagonal elements, we recall that the associated left (resp. right) orthonormal matrix polynomials with respect to the matrix measure $\Omega$ and $\Omega$ have the form $\left(U_{n} \Phi_{n}(z ; \Omega)\right)_{n \in \mathbb{N}}$ and $\left(V_{n} \Phi_{n}(z ; \Omega)\right)_{n \in \mathrm{N}}$ (resp. $\left(\Psi_{n}(z, \Omega) Y_{n}\right)_{n \in \mathbb{N}}$ and $\left.\left(\Psi_{n}(z ; \Omega) Z_{n}\right)_{n \in N}\right)\left(U_{n}, V_{n}, Y_{n}\right.$ and $Z_{n}$ are unitary matrices). Then the leading coefficients are related by

$$
\left\{\begin{array} { l } 
{ H _ { n } ( \Omega ) = U _ { n } L _ { n } ( \Omega ) } \\
{ H _ { n } ( \widetilde { \Omega } ) = V _ { n } L _ { n } ( \widetilde { \Omega } ) }
\end{array} \quad \text { and } \quad \left\{\begin{array}{l}
X_{n}(\Omega)=R_{n}(\Omega) Y_{n} \\
X_{n}(\widetilde{\Omega})=R_{n}(\widetilde{\Omega}) Z_{n}
\end{array}\right.\right.
$$

Therefore

$$
\begin{aligned}
& \left(H_{n}(\widetilde{\Omega}) H_{n}(\Omega)^{-1}\right) *=U_{n}\left(L_{n}(\widetilde{\Omega}) L_{n}(\Omega)^{-1}\right)^{*} V_{n}^{*}, \\
& \left(X_{n}(\Omega)^{-1} X_{n}(\widetilde{\Omega})\right)=Y_{n}^{*}\left(R_{n}(\Omega)^{-1} R_{n}(\widetilde{\Omega}) Z_{n} .\right.
\end{aligned}
$$

Setting $U_{n}=Y_{n}=I$, then the required relations (2.1) are satisfied. Now, we consider the sequences of orthonormal matrix polynomials $\left(T_{n} \Phi_{n}(z ; \widehat{\Omega})\right)_{n \in N}$ and $\left(\Psi_{n}(z ; \Omega) Q_{n}^{*}\right)_{n \in N}$, where $\left(T_{n}\right)_{n \in \mathbb{N}}$ and $\left(Q_{n}\right)_{n \in N}$ are unitary matrices given by the $Q R$ factorization of Francis and Kublanovskaja ${ }^{[5]}$ of $\left(L_{n}(\widetilde{\Omega}) L_{n}(\Omega)^{-1}\right)^{*}$ and $\left(R_{n}(\Omega)^{-1} R_{n}(\widetilde{\Omega})\right)$,

$$
\left\{\begin{array}{l}
\left(L_{n}(\widetilde{\Omega}) L_{n}(\Omega)^{-1}\right) *=R_{n} T_{n} \\
\left(R_{n}(\Omega)^{-1} R_{n}(\widetilde{\Omega})\right)=S_{n} Q_{n}
\end{array}\right.
$$

where $\left(R_{n}\right)_{n \in \mathbb{N}},\left(S_{n}\right)_{n \in \mathbb{N}}$ are lower triangular matrices with positive diagonal elements. In fact, let $\left(L_{n}(\widetilde{\Omega}) L_{n}(\Omega)^{-1}\right)$ and $\left(R_{n}(\Omega)^{-1} R_{n}(\widetilde{\Omega})\right)$ * be represented by

$$
\left(L_{n}(\widetilde{\Omega}) L_{n}(\Omega)^{-1}\right)=\widetilde{V}_{n} \widetilde{R}_{n} \text { and }\left(R_{n}(\Omega)^{-1} R_{n}(\widetilde{\Omega})\right)^{*}=\widetilde{Q}_{n} \widetilde{S}_{n},
$$

where $\widetilde{V}_{n}, \widetilde{Q}_{n}$, and $\widetilde{R}_{n} \widetilde{S}_{n}$ are, respectively, unitary matrices, and nonsingular upper triangular matrices, from the $Q R$ factorization. Then taking

$$
\left\{\begin{array} { l } 
{ R = \widetilde { R } _ { n } ^ { * } J _ { n } } \\
{ T _ { n } = J _ { n } ^ { * } \widetilde { V } _ { n } ^ { * } }
\end{array} \quad \text { and } \quad \left\{\begin{array}{l}
S_{n}=\widetilde{S}_{n}^{*} J_{n} \\
Q_{n}=J_{n}^{*} \widetilde{Q}_{n}^{*}
\end{array}\right.\right.
$$

where

$$
\left[J_{n}\right]_{i, j}= \begin{cases}\frac{\left[\tilde{R}_{n}\right]_{i, i}}{\left|\left[\tilde{R}_{n}\right]_{i, i}\right|} & \text { if } i=j \\ 0 & \text { otherwise }\end{cases}
$$


and

$$
\left[J_{n}\right]_{i, j}= \begin{cases}\frac{\left[\widetilde{S}_{n}\right]_{i, i}}{\left|\left[\widetilde{S}_{n}\right]_{i, i}\right|}, & \text { if } i=j \\ 0, & \text { otherwise, }\end{cases}
$$

the main diagonal of $R_{n}$ and $S_{n}$, are respectively

$$
\begin{aligned}
& \operatorname{diag}\left[R_{n}\right]=\left[\left|\left[\widetilde{R}_{n}\right]_{1,1}\right|,\left|\left[\widetilde{R}_{n}\right]_{2,2}\right|, \cdots,\left|\left[\widetilde{R}_{n}\right]_{p, p}\right|\right], \\
& \operatorname{diag}\left[S_{n}\right]=\left[\left|\left[\widetilde{S}_{n}\right]_{1,1}\right|,\left|\left[\widetilde{S}_{n}\right]_{2,2}\right|, \cdots,\left|\left[\widetilde{S}_{n}\right]_{p, p}\right|\right],
\end{aligned}
$$

and therefore (3.7) is given. Finally, using (3.6) and (3.7), we have

$$
\begin{aligned}
& \left(H_{n}(\widetilde{\Omega}) H_{n}(\Omega)^{-1}\right)^{*}=\left(L_{n}(\widetilde{\Omega}) L_{n}(\Omega)^{-1}\right)^{*} T_{n}^{*}=R_{n}, \\
& \left(X_{n}(\Omega)^{-1} X_{n}(\widetilde{\Omega})\right)=\left(R_{n}(\Omega)^{-1} R_{n}(\widetilde{\Omega})\right) Q_{n}^{*}=S_{n} .
\end{aligned}
$$

Hence, $\left(H_{n}(\Omega) H_{n}(\Omega)^{-1}\right)_{n \in \mathbb{N}}$ and $\left(X_{n}(\Omega)^{-1} X_{n}(\Omega)\right)_{n \in \mathbb{N}}$ are lower triangular matrices with positive diagonal elements.

Now, we are ready to prove the main theorem in this section.

Theorem 3. 4. Let $\Omega$ and $\Omega$, be two matrix measures related by (3.1). If the Szegö condition

$$
\log \operatorname{det} W \in L^{1}(T)
$$

holds, then

$$
\lim _{n \rightarrow \infty}\left(L_{n}(\widetilde{\Omega}) L_{n}(\Omega)^{-1}\right)=\lim _{n \rightarrow \infty}\left(R_{n}(\Omega)^{-1} R_{n}(\widetilde{\Omega})\right)=\frac{1}{|w|} I
$$

when $|w|>1$.

First, we start with the following lemma.

Lemma 3.5. Under the hypothesis (3.8), we have

$$
\lim _{n \rightarrow \infty} \Phi_{n}(w ; \Omega)^{-*}=\lim _{n \rightarrow \infty} \Psi_{n}(w ; \Omega)^{-*}=0,
$$

when $w$ is outside the closed unit disk.

Proof. From (2.3) and (2.4), we have

$$
\begin{array}{ll}
\Phi_{n}\left(\frac{1}{w}, \Omega\right)^{-*}=M_{n}^{*} A_{n}(w)^{-1} w^{n}, & \text { with } M_{n}^{*} M_{n}=A_{n}(0)^{-1} \\
\Psi_{n}\left(\frac{1}{w_{w}}, \Omega\right)^{-*}=B_{n}(w)^{-1} N_{n}^{-*} w^{n}, & \text { with } N_{n} N_{n}^{*}=B_{n}(0)^{-1},
\end{array}
$$

since

$$
\begin{aligned}
& \left\|M_{n}^{-*}\right\|_{2}^{2}=\mu_{\left(N_{n}^{-1} M_{n}^{-*}\right)}=\mu_{\left(A_{n}(0)\right)}, \\
& \left\|N_{n}^{-*}\right\|_{2}^{2}=\mu_{\left(N_{n}^{-1} N_{n}^{-*}\right)}=\mu_{\left(N_{n}^{-} \cdot N_{n}^{-1}\right)}=\mu_{\left(B_{n}(0)\right)},
\end{aligned}
$$

where $\|\cdot\|_{2}$ is the spectral norm and $\mu_{(\cdot)}$ is the spectral radius, then from (2.18) and Theorem 2.1 we have 


$$
\begin{aligned}
& \left\|M_{n}^{-*}\right\| \frac{2}{2}<\infty, \quad\left\|N_{n}^{-*}\right\| \frac{2}{2}<\infty, \\
& \left\|A_{n}(w)^{-1}\right\|_{2}^{2}<\infty \text { and }\left\|B_{n}(w)^{-1}\right\| \frac{2}{2}<\infty \quad \text { for }|w|<1 .
\end{aligned}
$$

Hence

$$
\begin{array}{ll}
\left\|\Phi_{n}\left(\frac{1}{w} ; \Omega\right)^{-*}\right\|_{2} \leqslant\left\|M_{n}^{-*}\right\|_{2} \cdot\left\|A_{n}(w)^{-1}\right\|_{2} \cdot|w|^{n}, & |w|<1, \\
\left\|\Psi_{n}\left(\frac{1}{w} ; \Omega\right)^{-*}\right\|_{2} \leqslant\left\|N_{n}^{-*}\right\|_{2} \cdot\left\|B_{n}(w)^{-1}\right\|_{2} \cdot|w|^{n}, & |w|<1
\end{array}
$$

which yield

$$
\lim _{n \rightarrow \infty} \Phi_{n}\left(w_{3} \Omega\right)^{-*}=\lim _{n \rightarrow \infty} \Psi_{n}\left(w_{3} \Omega\right)^{-\cdot}=0, \quad \text { for }|w|>1 .
$$

Proof of Theorem 3.4. From (2.15) and (2.14) we have

$$
\begin{aligned}
& \mathscr{K}_{n}(w, w ; \Omega)=\frac{1}{|w|^{2}-1}\left\{|w|^{2} \Phi_{n}(w ; \Omega) * \Phi_{n}(w ; \Omega)-\hat{\Psi}_{n}(w ; \Omega) * \hat{\Psi}_{n}(w ; \Omega)\right\}, \\
& \mathscr{K}_{n}(w, w ; \Omega)=\frac{1}{|w|^{2}-1}\left\{|w|^{2} \Psi_{n}(w ; \Omega) \Psi_{n}(w ; \Omega)^{*}-\hat{\Phi}_{n}(w ; \Omega) \Phi_{n}(w ; \Omega)^{*}\right\} .
\end{aligned}
$$

Then

$$
\begin{aligned}
& \Phi_{n}(w ; \Omega)^{-"} \mathscr{K}_{n}(w, w ; \Omega) \Phi_{n}(w ; \Omega)^{-1} \\
& \quad=\frac{1}{|w|^{2}-1}\left\{|w|^{2} I-\left[\hat{\Psi}_{n}(w ; \Omega) \Phi_{n}(w ; \Omega)^{-1}\right] \cdot\left[\hat{\Psi}_{n}(w ; \Omega) \Phi_{n}(w ; \Omega)^{-1}\right]\right\}
\end{aligned}
$$

and

$$
\begin{aligned}
& \Psi_{n}(w ; \Omega)^{-1} \mathscr{Z}_{n}(w, w ; \Omega) \Psi_{n}(w ; \Omega)^{-*} \\
& \quad=\frac{1}{|w|^{2}-1}\left\{|w|^{2} I-\left[\Psi_{n}(w ; \Omega)^{-1} \Phi_{n}(w ; \Omega)\right]\left[\Psi_{n}(w ; \Omega)^{-1} \Phi_{n}(w ; \Omega)\right]^{*}\right\} .
\end{aligned}
$$

Using Theorem 2.2 , we get

$$
\begin{aligned}
& \lim _{n \rightarrow \infty} \Phi_{n}(w ; \Omega)^{-*} \mathscr{K}_{n}(w, w ; \Omega) \Phi_{n}(w ; \Omega)^{-1} \\
& =\lim _{n \rightarrow \infty} \Psi_{n}(w ; \Omega)^{-1} \mathscr{K}_{n}(w, w ; \Omega) \Psi_{n}(w ; \Omega)^{-*}=\frac{|w|^{2}}{|w|^{2}-1} I,
\end{aligned}
$$

and using Lemma 3.5 we get

$$
\lim _{n \rightarrow \infty} \Phi_{n}(w ; \Omega)^{-*} \mathscr{M}^{-1} \Phi_{n}(w ; \Omega)^{-1}=\lim _{n \rightarrow \infty} \Psi_{n}(w ; \Omega)^{-1} \mathscr{M}^{-1} \Psi_{n}(w ; \Omega)^{-*}=0 .
$$

Finally, from (3.10), (3.11) and Proposition 3.2, we have

$$
\begin{aligned}
\lim _{n \rightarrow \infty}\left(L_{n}(\widetilde{\Omega}) L_{n}(\Omega)^{-1}\right) \cdot\left(L_{n}(\widetilde{\Omega}) L_{n}(\Omega)^{-1}\right) \\
\quad=\lim _{n \rightarrow \infty}\left(R_{n}(\Omega)^{-1} R_{n}(\widetilde{\Omega})\right)\left(R_{n}(\Omega)^{-1} R_{n}(\widetilde{\Omega})\right)^{*}=\frac{1}{|w|^{2}} I .
\end{aligned}
$$

Since $\mathscr{M}_{\mathscr{K}_{n}}(w, w ; \Omega)^{*}$ and $\mathscr{X}_{n}(w, w ; \Omega)^{*}$ are positive definite, then using Proposition 3.2, we have

$$
\mathscr{L}_{n}(w)=\left(L_{n}(\widetilde{\Omega}) L_{n}(\Omega)^{-1}\right) \cdot\left(L_{n}(\widetilde{\Omega}) L_{n}(\Omega)^{-1}\right)<I
$$




$$
\mathscr{R}_{n}(w)=\left(R_{n}(\Omega)^{-1} R_{n}(\widetilde{\Omega})\right)\left(R_{n}(\Omega)^{-1} R_{n}(\widetilde{\Omega})\right)^{*}<I .
$$

Let $\|\cdot\|_{\varepsilon}$ be the Frobenius norm, then

$$
\begin{aligned}
& \left\|L_{n}(\widetilde{\Omega}) L_{n}(\Omega)^{-1}\right\|_{E}^{2}=\operatorname{tr} \mathscr{L}_{n}(w) \leqslant p \mu_{\left(\mathscr{X}_{n}(w)\right)}=p\left\|L_{n}(\widetilde{\Omega}) L_{n}(\Omega)^{-1}\right\|_{2}^{2}<p, \\
& \left\|\left(R_{n}(\widetilde{\Omega})^{-1} R_{n}(\widetilde{\Omega})\right)^{*}\right\|_{E}^{2}=\operatorname{tr} \mathscr{R}_{n}(w)<p .
\end{aligned}
$$

Let $\left(n_{v}\right)_{v \in N}$ and $\left(n_{v}\right)_{n \in N}$ be two increasing sequences of positive integer numbers such that the limits

$$
\begin{aligned}
& \mathscr{A}=\lim _{\nu \rightarrow \infty}\left(L_{n_{\nu}}(\widetilde{\Omega}) L_{n_{\nu}}(\Omega)^{-1}\right)^{*}, \\
& \mathscr{B}=\lim _{\nu \rightarrow \infty}\left(R_{n_{\nu}}(\Omega)^{-1} R_{n_{v}}(\widetilde{\Omega})\right)
\end{aligned}
$$

exist. Then from (3.12), we have

$$
\mathscr{A} \mathscr{A}^{*}=\mathscr{B} \mathscr{B}^{*}=\frac{1}{|w|^{2}} I \text {. }
$$

Since $\frac{1}{|w|^{2}} I$ is positive definite, and $\mathscr{A}, \mathscr{B}$ have positive diagonal elements, then using the Cholesky factorization, the representation (3.13) is unique and then $\left(L_{n}\left(\Omega_{)}\right) L_{n}\right.$ $\left.(\Omega)^{-1}\right)^{*}$ and $\left(R_{n}(\Omega)^{-1} R_{n}(\widetilde{\Omega})\right)$ have no subsequences that converge to a limit other than $\frac{1}{|w|} I$. Hence (3. 9) holds.

Corollary 3.6. Let $\Omega$ be a matrix-valued measure supported on $\mathrm{T}$. We assume that the Szegö condition (3.8) holds. Then

$$
\begin{aligned}
& \lim _{n \rightarrow \infty} \Phi_{n}(w ; \Omega)^{-*} \mathscr{K}_{n}(w, w ; \Omega) \Phi_{n}(w ; \Omega)^{-1} \\
& =\lim _{n \rightarrow \infty} \Psi_{n}(w ; \Omega)^{-1} \mathscr{Z}_{n}(w, w ; \Omega) \Psi_{n}(w ; \Omega)^{-\cdot}=\frac{|w|^{2}}{|w|^{2}-1} I,
\end{aligned}
$$

for $w$ outside the closed unit disk.

\section{Relative Asymptotics for Orthonormal Matrix Polynomials}

We give now the asymptotic behavior for the ratio and the product of two sequences of orthonormal matrix polynomials associated respectively, to a matrix measure supported on the unit circle and its perturbation by the addition of a Dirac matrix measure, supported at a point outside of the closed unit disk.

Theorem 4. 1. Let $\left(\Phi_{n}(z ; \Omega)\right)_{n \in \mathbb{N}}$ and $\left(\Phi_{n}(z ; \Omega)\right)_{n \in N}\left(\operatorname{resp} .\left(\Psi_{n}(z ; \Omega)\right)_{n \in N}\right.$ and $\left(\Psi_{n}(z\right.$; $\widetilde{\Omega}))_{n \in \mathbb{N}}$ ) be sequences of left (resp. right) orthonormal matrix polynomials with respect to the matrix measure $\Omega$ and $\Omega$, related by

$$
\widehat{\Omega}(z)=\Omega(z)+\mathscr{M} \delta(z-w) ; \quad|w|>1 .
$$




\section{If the Szegö condition}

$$
\log \operatorname{det} W \in L^{1}(T)
$$

holds, then

$$
\begin{aligned}
& \lim _{n \rightarrow \infty} \Phi_{n}(z ; \Omega) \Phi_{n}(z ; \Omega)^{-1} \\
& \quad=\lim _{n \rightarrow \infty} \Psi_{n}(z ; \Omega)^{-1} \Psi_{n}(z ; \Omega)=\frac{\bar{w}}{|w|}\left(\frac{w-z}{1-z w}\right) I \quad \text { for }|z| \geqslant 1 .
\end{aligned}
$$

Proof. Multiplying in the first part of Lemma 3.1 by $\Phi_{n}(z ; \Omega)^{-1}$, we have

$$
\begin{gathered}
\Phi_{n}(z ; \widetilde{\Omega}) \Phi_{n}(z ; \Omega)^{-1}=\left(L_{n}(\Omega) L_{n}(\Omega)^{-1}\right)^{-*}\{I- \\
\left.\Phi_{n}(w ; \Omega)\left[I+\mathscr{M} \mathscr{K}_{n}(w, w ; \Omega)\right]^{-1} \mathscr{M} \mathscr{K}_{n}(z, w ; \Omega) " \Phi_{n}(z ; \Omega)^{-1}\right\} .
\end{gathered}
$$

But using (2.13), we get

$$
\begin{aligned}
& \Phi_{n}(w ; \Omega)^{-*} \mathscr{K}_{n}(z, w ; \Omega) * \Phi_{n}(z ; \Omega)^{-1} \\
& =\frac{1}{1-z \bar{w}}\left\{\Phi_{n}(w ; \Omega)^{-*} \dot{\Psi}_{n}(w ; \Omega) * \Psi_{n}(z ; \Omega) \Phi_{n}(z ; \Omega)^{-1}-z \bar{w} I\right\},
\end{aligned}
$$

and from Proposition 3.2,

$$
\begin{gathered}
\Phi_{n}(w ; \Omega)\left[I+\mathscr{M} \mathscr{K}_{n}\left(w, w_{n} \Omega\right)\right]^{-1} \mathscr{M} \Phi_{n}\left(w_{3} \Omega\right)^{*} \\
=I-\left(L_{n}(\widetilde{\Omega}) L_{n}(\Omega)^{-1}\right) \cdot\left(L_{n}(\widetilde{\Omega}) L_{n}(\Omega)^{-1}\right) .
\end{gathered}
$$

Then

$$
\begin{aligned}
& \Phi_{n}(z ; \widetilde{\Omega}) \Phi_{n}(z ; \Omega)^{-1}=\left(L_{n}(\widetilde{\Omega}) L_{n}(\Omega)^{-1}\right)^{-*}\{I- \\
& {\left[I-\left(L_{n}(\widetilde{\Omega}) L_{n}(\Omega)^{-1}\right)^{*}\left(L_{n}(\widetilde{\Omega}) L_{n}(\Omega)^{-1}\right)\right] \cdot } \\
&\left.\frac{1}{1-z \bar{w}}\left[\Phi_{n}(w ; \Omega)^{-*} \hat{\Psi}_{n}\left(w_{3} \Omega\right)^{*} \hat{\Psi}_{n}(z ; \Omega) \Phi_{n}(z, \Omega)^{-1}-z \bar{w} I\right]\right\} \\
&=\left(L_{n}(\widetilde{\Omega}) L_{n}(\Omega)^{-1}\right)^{-*}-\frac{1}{1-z \bar{w}}\left[\left(L_{n}(\Omega) L_{n}(\Omega)^{-1}\right)^{-*}-\right. \\
&(\left.\left.L_{n}(\Omega) L_{n}(\Omega)^{-1}\right)\right]\left[\left(\hat{\Psi}_{n}(w ; \Omega) \Phi_{n}(w ; \Omega)^{-1}\right) *\left(\hat{\Psi}_{n}(z ; \Omega) \Phi_{n}(z ; \Omega)^{-1}\right)-z \bar{w} I\right] .
\end{aligned}
$$

From Theorem 2. 2, we have

$$
\lim _{n \rightarrow \infty} \Psi_{n}(z ; \Omega) \Phi_{n}(z ; \Omega)^{-1}=0, \quad \text { for }|z|>1 .
$$

From (2.13), when we take $z=\xi \in T$ we get the identity

$$
\hat{\Psi}_{n}(z ; \Omega) * \hat{\Psi}_{n}(z ; \Omega)=\Phi_{n}(z ; \Omega) * \Phi_{n}(z ; \Omega) \text {. }
$$

This means that

$$
\hat{\Psi}_{n}(z, \Omega) \Phi_{n}(z ; \Omega)^{-1}=\left(\Psi_{n}(z ; \Omega) \Phi_{n}(z ; \Omega)^{-1}\right)^{-*} \text { for } z \in \mathrm{T} .
$$

Thus, the rational matrix function $\hat{\Psi}_{n}\left(z_{;} \Omega\right) \Phi_{n}\left(z_{3} \Omega\right)^{-1}$ is a unitary matrix when $z$ is on the unit circle, and then $\left\|\hat{\Psi}_{n}(z ; \Omega) * \Psi_{n}(z ; \Omega)\right\|_{2}=1$. Therefore,

$$
\lim _{n \rightarrow \infty}\left(\dot{\Psi}_{n}(w ; \Omega) \Phi_{n}(w ; \Omega)^{-1}\right) *\left(\hat{\Psi}_{n}(z ; \Omega) \Phi_{n}(z ; \Omega)^{-1}\right)=0
$$

for $|w|>1$ and $|z| \geqslant 1$. 
Hence,

$$
\lim _{n \rightarrow \infty} \Phi_{n}(z ; \widetilde{\Omega}) \Phi_{n}(z ; \Omega)^{-1}=|w| I+\frac{z \bar{w}}{1-z \bar{w}}\left(|w|-\frac{1}{|w|}\right) I=\frac{\bar{w}}{|w|}\left(\frac{w-z}{1-z \bar{w}}\right) I .
$$

To prove the second part of (4.2), we take into account that the following assertions are true

1. $\Psi_{n}(z ; \Omega)^{-1} \mathscr{X}_{n}(z, w ; \Omega) * \Psi_{n}(w ; \Omega)^{-*}$

$$
=\frac{1}{z \bar{w}-1}\left\{z \bar{w} I-\left[\Psi_{n}(z, \Omega)^{-1} \phi_{n}(z ; \Omega)\right]\left[\Psi_{n}(w ; \Omega)^{-1} \phi_{n}(w, \Omega)\right]^{*}\right\},
$$

2. $\Psi_{n}(w ; \Omega) * \mathscr{M}\left[I+\mathscr{H}_{n}(z, w ; \Omega) * \mathscr{M}\right]^{-1} \Psi_{n}(w ; \Omega)$

$$
=I-\left(R_{n}(\Omega)^{-1} R_{n}(\widetilde{\Omega})\right)\left(R_{n}(\Omega)^{-1} R_{n}(\widetilde{\Omega})\right)^{*},
$$

3. $\Psi_{n}(z ; \Omega)^{-1} \Psi_{n}(z ; \Pi)$

$$
\begin{aligned}
= & \left\{I-\Psi_{n}(z ; \Omega)^{-1} \mathscr{E}_{n}(z, w ; \Omega) * \mathscr{M}[I+\right. \\
& \left.\mathscr{H}(w, w ; \Omega) \mathscr{M}]^{-1} \Psi_{n}(w ; \Omega)\right\}\left(R_{n}(\Omega)^{-1} R_{n}(\Omega)\right)^{-*} .
\end{aligned}
$$

Then using (2.21), (3.9) and the fact that $\left(\Psi_{n}(z, \Omega)^{-1} \Phi_{n}(z, \Omega)\right)_{n \in N}$ are unitary matrices when $z \in T$, we get the second part of (4.2).

Theorem 4. 2. Let $\Omega$ and $\Omega$ be two matrix measures which are related by

$$
\Pi(z)=\Omega(z)+\mathscr{M} \delta(z-w) ; \quad|w|>1 \text { and } \mathscr{M}=I .
$$

We assume that the Szegö condition (4.1) holds, then

$$
\begin{aligned}
& \lim _{n \rightarrow \infty} \Phi_{n}(w ; \Omega) \Phi_{n}(w ; \Omega) * \\
& \quad=\lim _{n \rightarrow \infty} \Psi_{n}(w ; \Omega) * \Psi_{n}(w ; \widetilde{\Omega})=\frac{|w|^{2}-1}{|w|} I .
\end{aligned}
$$

Proof. If we multiply in the first formula of Lemma 3.1 by $\Phi_{n}\left(z_{3} \Omega\right)$ ", and evaluating the resulting expression at $z=w$, then

$$
\begin{gathered}
\Phi_{n}(w ; \Omega) \Phi_{n}(w ; \Omega)^{*}=\left(L_{n}(\Omega) L_{n}(\Omega)^{-1}\right)^{* *}\left\{\Phi_{n}(w ; \Omega) \Phi_{n}(w ; \Omega) *-\right. \\
\left.\Phi_{n}(w ; \Omega)\left[I+\mathscr{M} \mathscr{K}_{n}(w, w ; \Omega)\right]^{-1} \mathscr{M} \mathscr{K}_{n}(w, w ; \Omega) \Phi_{n}(w ; \Omega)^{*}\right\} .
\end{gathered}
$$

Since

$$
\begin{aligned}
\Phi_{n}(w ; \Omega) \Phi_{n}(w ; \Omega) *= & \Phi_{n}(w ; \Omega)\left[I+\mathscr{M} \mathscr{K}_{n}(w, w ; \Omega)\right]^{-1} \mathscr{M} \mathscr{K}_{n}(w, w ; \Omega) \Phi_{n}(w ; \Omega)^{*} \\
& +\Phi_{n}(w ; \Omega)\left[I+\mathscr{M} \mathscr{K}_{n}(w, w ; \Omega)\right]^{-1} \Phi_{n}(w ; \Omega)^{*},
\end{aligned}
$$

then

$$
\begin{aligned}
& \Phi_{n}(w ; \widetilde{\Omega}) \Phi_{n}(w ; \Omega) * \\
&=\left(L_{n}(\widetilde{\Omega}) L_{n}(\Omega)^{-1}\right)^{-*} \Phi_{n}(w ; \Omega)\left[I+\mathscr{M} \mathscr{K}_{n}(w, w ; \Omega)\right]^{-1} \Phi_{n}(w ; \Omega) * \\
&=\left(L_{n}(\widetilde{\Omega}) L_{n}(\Omega)^{-1}\right)^{-*}\left\{\Phi_{n}(w ; \Omega)^{-*} \Phi_{n}(w ; \Omega)^{-1}+\right. \\
&\left.\Phi_{n}(w ; \Omega)^{-*} \mathscr{M} \mathscr{K}_{n}(w, w ; \Omega) \Phi_{n}(w ; \Omega)^{-1}\right\}^{-1} .
\end{aligned}
$$

Since $\mathscr{M}=I$ then using (2.13), we get 


$$
\begin{aligned}
& \Phi_{n}(w ; \Omega)^{-*} \mathscr{K}_{n}(w, w ; \Omega) \Phi_{n}(w ; \Omega)^{-1} \\
& \quad=\frac{1}{1-|w|^{2}}\left\{\Phi_{n}(w ; \Omega)^{-*} \hat{\Psi}_{n}(w ; \Omega) * \hat{\Psi}_{n}(w ; \Omega) \Phi_{n}(w ; \Omega)^{-1}-|w|^{2} I\right\} .
\end{aligned}
$$

From Lemma 3.5 and Theorem 2.2, we have

$$
\begin{aligned}
& \lim _{n \rightarrow \infty} \Phi_{n}(w ; \Omega)^{-*} \Phi_{n}(w ; \Omega)^{-1}=0, \\
& \lim _{n \rightarrow \infty} \Phi_{n}(w ; \Omega)^{-*} \mathscr{M} \mathscr{K}_{n}(w, w ; \Omega) \Phi_{n}(w ; \Omega)^{-1}=\frac{|w|^{2}}{|w|^{2}-1} I .
\end{aligned}
$$

Then using Theorem 3.4 , we get

$$
\lim _{n \rightarrow \infty} \Phi_{n}(w ; \Omega) \Phi_{n}(w ; \Omega)^{*}=|w|\left\{\frac{|w|^{2}}{|w|^{2}-1} I\right\}^{-1}=\frac{|w|^{2}-1}{|w|} I .
$$

Finally, in a similar way, we prove the second part of (4.3).

Acknowledgement. The work of the second author was supported by Direction General de Ensenanza Superior (DGES) of Spain under grant PB96-0120-CO3-01 and INTAS Project INTAS93-0219 Ext.

\section{References}

[1] Aptekarev, A. I. and Nikishin, E. M. , The Scattering Problem for a Discrete Sturm-Liouville Operator, Mat. USSR Sb. , 49,1984, 325-355.

[2] Delsarte, Ph., Genin, Y. V. and Kamp, Y. G. , Orthogonal Polynomial Matrices on the Unit Circle, IEEE Trans. Circuits and Systems $25,3,1978,149-160$.

[3] Geronimo, J. S. , Matrix Orthogonal Polynomials on the Unit Circle, J. Math. Phys. , 22,7, $1981,1359-1365$.

[4] Gohberg, I. , Lancaster, P. and Rodman, L., Matrix Polynomials, Academic Press, New York, 1982.

[5] Horn, R. A. and Johnson, C. A. , Topics in Matrix Analysis, Cambridge University Press, Cambridge, 1991.

[6] Marcellán, F. and Rodríguez, González, I. , A Class of Matrix Orthogonal Polynomials on the Unit Circle, Linear Algebra and Appl. , 121, 1989, 233-241.

[7] Mignolet, M. P. , Matrix Polynomials Orthogonal on the Unit Circle and Accuracy of Autoregressive Models, J. Comp. Appl. Math. , 62,1995, 229-238.

[8] Peherstorfer, F. and Steinbauer, R. , Mass-Points of Positive Borel Measures, Manuscript, 1998.

[9] Rodman, L. , Orthogonal Matrix Polynomials, In Orthogonal Polynomials : Theory and Prac- 
tice (P. Nevai, ed. ), NATO Asi Series C, Vol. 294, Kluwer, Dordrecht, 1990, 345-362.

[10] Rosemberg, M. , The Square-Integrability of Matrix-Valued Functions with Respect to a NonNegative Hermitian Measure, Duke Math. J. , 31,1964, 291-298.

[11] Van Assche, W. , Orthogonal Polynomials in the Complex Plane and on the Real Line, In Special Functions, $q$-Series and Related Topics, Fields Institute Communications, Vol. 14, Amer. Math. Soc. , Providence Rhode Island, 1997, 211-245.

[12] Rakhmanov's Theorems for Orthogonal Matrix Polynomials on the Unit Circle, Manuscript, 1998.

[13] Yakhlef, H. O. , Marcellán, F. and Pinñar, M. , Relative Asymptotics for Orthogonal Matrix Polynomials with Convergent Recurrence Coefficients, Manuscript, 1998.

[14] Youla D. C. and Kazanjian, N. N. , Bauer-Type Factorization of Positive Matrices and the Theory of Matrix Polynomials Orthogonal on the Unit Circle, IEEE Trans. Circuits and Systems $25: 2,1978,57-69$.

Hossain O. Yakhlef

Departamento De Matemática Aplicada

Universidad De Granada

C/Severo Ochoa

S/N 18071 Granada-Spain

E-mail address:houlad@goliat.ugr.es

Francisco Marcellán

Departamento de Matemáticas

Universidad Carlos I de Madrid

C/Butarque, 15, 28911 Leganés-Spain

E-mail address : pacomarc@ing.uc3m.es 